\title{
Spatial and Temporal Characteristics of EAS with Delayed Particles
}

\author{
Rashid Beisembaev, Elena Beisembaeva, Oleg Dalkarov, Vasily Mossunov, \\ Vladimir Ryabov, Sergei Shaulov, Marina Vildanova, Valeriy Zhukov \\ P. N. Lebedev Physical Institute of the Russian Academy of Sciences \\ 53 Leninskiy avenue, Moscow, Russia \\ Dmitriy Beznosko ${ }^{1}$ \\ Harvard University \\ 1 Oxford Street, Cambridge, MA, USA \\ E-mail: dmitriy.beznosko@dozory.us
}

\section{Kanat Baigarin}

Nazarbayev University

53 Kabanbai Batyr ave, Astana, Kazakhstan

\section{Turlan Sadykov}

Satbayev University, Institute of Physics and Technology

Ibragimova str. 11, Almaty, Kazakhstan

The Extensive Air Showers (EAS) with delayed particles have been detected by the Horizon-10T experiment, located at the elevation of $3346 \mathrm{~m}$ above sea level near the city of Almaty, Republic of Kazakhstan. Among these EAS with delayed particles there were events that had signals with two distinct pulses (maximums or modes). Such pulses we call bimodal, and showers - bimodal events.

This article presents the study of the properties of only bimodal events and comparison of these properties with the EAS that were simulated using the CORSIKA software package. This thorough comparison has shown that bimodal events cannot be explained only by known physical processes taking place in electron-hadron showers.

36th International Cosmic Ray Conference -ICRC2019-

July 24th - August 1st, 2019

Madison, WI, U.S.A.

\section{${ }^{1}$ Speaker}




\section{Introduction}

An important part towards the understanding of the nature of cosmic rays is the study of the Extensive Air Showers (EAS) with delayed particles. Jelley and Whitehouse were the first ones studying these type of EAS in 1953 [1]. Later, EAS exhibiting the unusual time structures were studied by independent experiments such as [2-5] and others. All these studies concluded that EAS with delayed particles cannot be explained using known physical processes.

For the detailed studies of these EAS with delayed particles, the Horizon-10T [6] (H10T), innovative detector system designed with ns-level time resolution using scintillator-based detectors was constructed at Tien Shan High Altitude Science Station located at $\sim 3340 \mathrm{~m}$ above the sea level near the city of Almaty, Kazakhstan. Figure 1 shows the aerial view of H10T and location of the detection points. The distance from the detector system center to each detection point is given in Table 1. Table 2 has the H10T acceptance and the expected EAS rate as N/t depending on the energy of the primary particle $\mathrm{E}_{0}$.

In this article, we present the analysis result of the experimental data from H10T from the period from Feb. 15, 2018 to May 12, 2018.

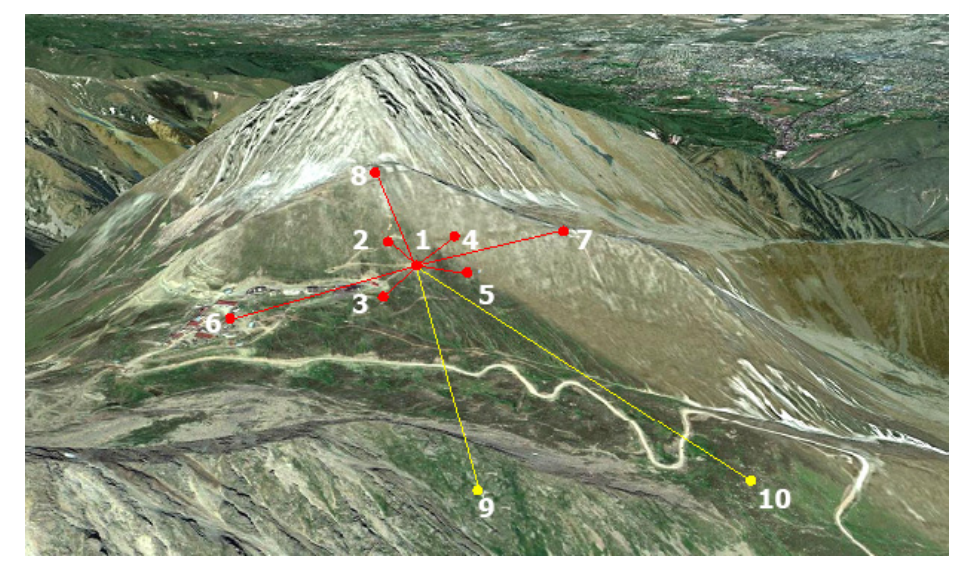

Figure 1: Aerial view and geometry of H10T detector.

Table 1: Distance R of each detection point from center.

\begin{tabular}{|c|c|c|c|c|c|c|c|c|c|c|}
\hline Point \# & 1 & 2 & 3 & 4 & 5 & 6 & 7 & 8 & 9 & 10 \\
\hline R, $\mathrm{m}$ & 0 & 133 & 148 & 155 & 194 & 454 & 393 & 367 & 594 & 1000 \\
\hline
\end{tabular}

Table 2: Detector system acceptance and event rate.

\begin{tabular}{|c|c|c|c|c|c|c|}
\hline $\mathrm{E}_{0}[\mathrm{eV}]$ & $10^{16}$ & $2 \cdot 10^{16}$ & $5 \cdot 10^{16}$ & $10^{17}$ & $2 \cdot 10^{17}$ & $10^{18}$ \\
\hline$\Gamma\left[\mathrm{km}^{2} \mathrm{sr}\right]$ & 0.38 & 0.72 & 0.97 & 1.52 & 2.72 & 6.31 \\
\hline $\mathrm{N} / \mathrm{t}[\mathrm{event} / \mathrm{h}]$ & 25.60 & 7.98 & 2.25 & 1.04 & 0.52 & 0.06 \\
\hline
\end{tabular}

\section{EAS Representation as Electron-Hardon Shower}

A conceptual model of EAS has been suggested in 1948 by G. T. Zatsepin [7] that views EAS as electron-nuclear showers from a primary ultra-high energy particle. Nowadays, electronnuclear showers are more correctly called electron-hadron (EHS). Hadrons form the core of EAS, electron-photon component is secondary. 
The representation of EAS as EHS is realized in the CORSIKA [8] simulation package. Let as review the properties of simulated EHS at the observation level that is a horizontal plane at the altitude of 3346 above sea level.

The most dominant species of charged particles in EHS are electrons and muons. In showers that are simulated with zenith angle from zero to $20^{\circ}$, electrons dominate by about factor of hundred; for showers with zenith angles $70^{\circ}$ and larger - muons start to dominate.

All ultra-relativistic EHS particles that reach observation level are distributed in space in the shape of the disk. Figure 2 shows the cross-section of a simulated EAS (e.g. EHS) disk from the primary proton with energy $E_{0}=2 \cdot 10^{15} \mathrm{eV}$ arriving vertically. In this figure, $\mathrm{Z}$ is the EAS axis, and $\mathrm{R}_{0}$ is the distance from $\mathrm{Z}$-axis in the observation plane. The figure represents the positions of all simulated particles in the disk when the first one arrives at the observation level. Muons are in red and electrons are shown as blue dots.

The $\sim 20 \%$ of particles above the green line are showers from gamma particles that were born from $\pi^{0}$ decays near the observation plane so they do not carry significant information about overall EHS development. For further analysis, we will consider the time that the first $80 \%$ of particles pass through a detector as the width of the disk and denote it as $\tau_{0.8}$.

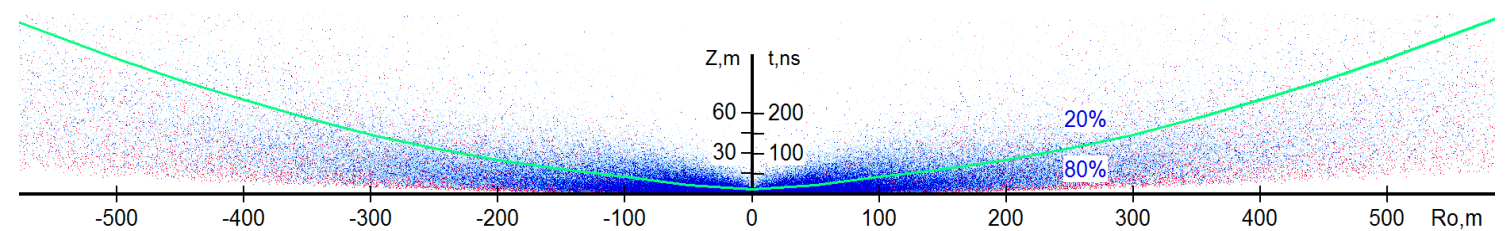

Figure 2: Particle distribution in simulated EAS (EHS) disk vs. distance from EAS axis.

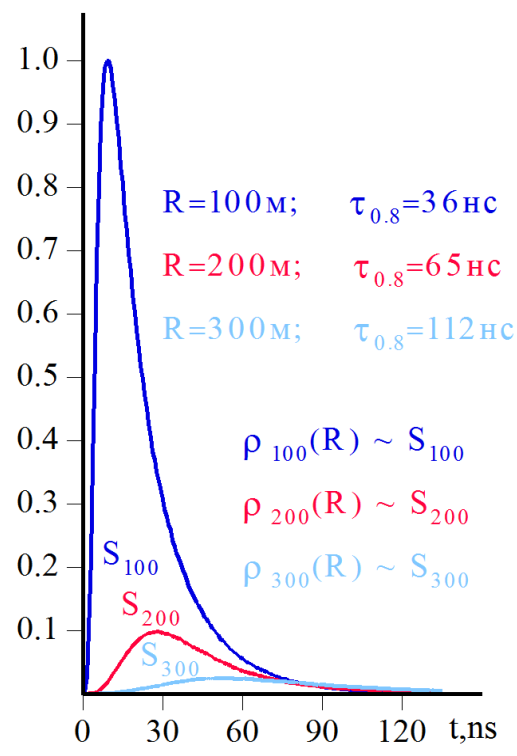

Figure 3: Detector pulses at different distances from EAS axis.

The pulses in the detectors are presented in Figure 3. These identical detectors are at $\mathrm{R}=$ $100 \mathrm{~m}$ (blue line), $200 \mathrm{~m}$ (red) and $300 \mathrm{~m}$ (light blue). The pulse areas are proportional to number of particles detected and to particle density $\rho$ at that distance. The pulse area decreases with $\mathrm{R}$ and the duration increases. 


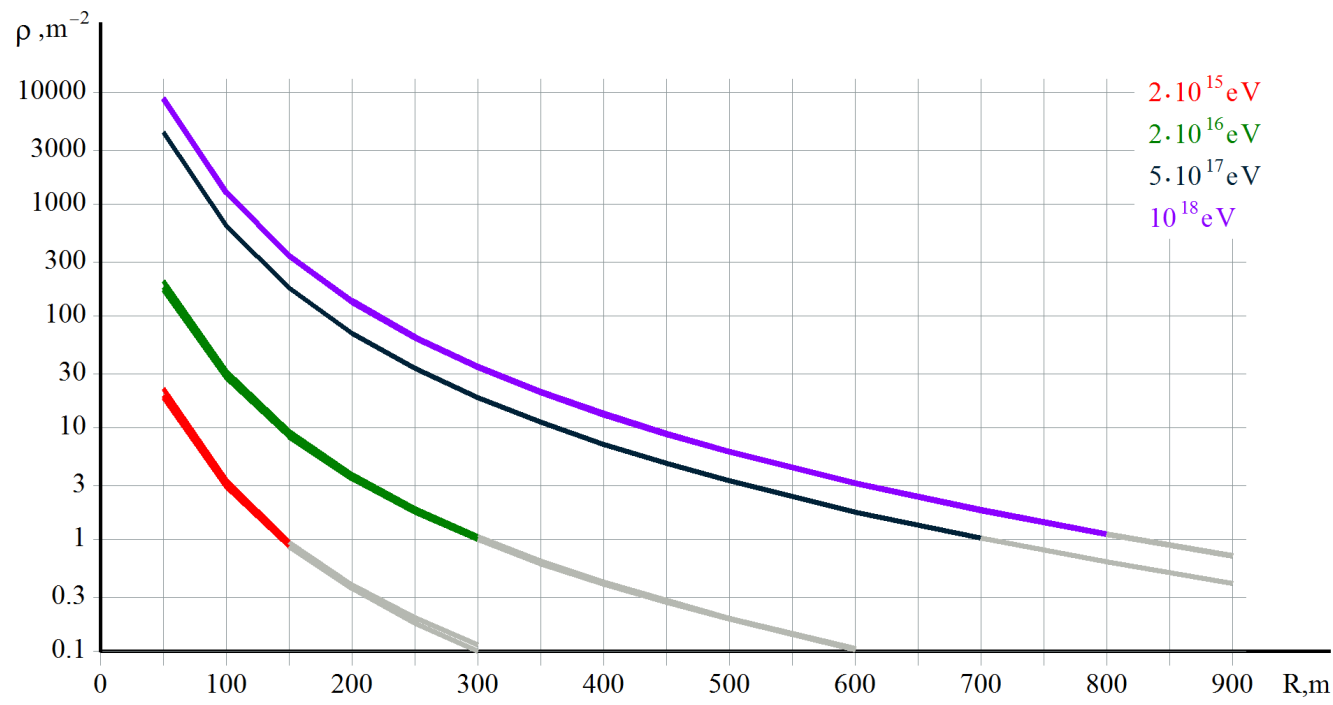

Figure 4: Particle density distribution in simulated EAS disk vs. distance from axis for different $E_{0}$.

The dependence of particle density in the simulated EAS with energy of the primary and the distance from the axis is shown in Figure 4. The plots are made for primary protons with four different $\mathrm{E}_{0}$ : the lowest line in red represents the $E_{0}=2 \cdot 10^{15} \mathrm{eV}$, next in green $-2 \cdot 10^{16} \mathrm{eV}$, second from the top in black $-5 \cdot 10^{17} \mathrm{eV}$, and the topmost in violet $-10^{18} \mathrm{eV}$. The gray color means that the particle density drops below one particle $/ \mathrm{m}^{2}$. The functional approximation of density dependence on distance from axis $\rho(R)$ is typically called a spatial distribution function. Additionally, particle density has a close to linear dependence on $\mathrm{E}_{0}$.

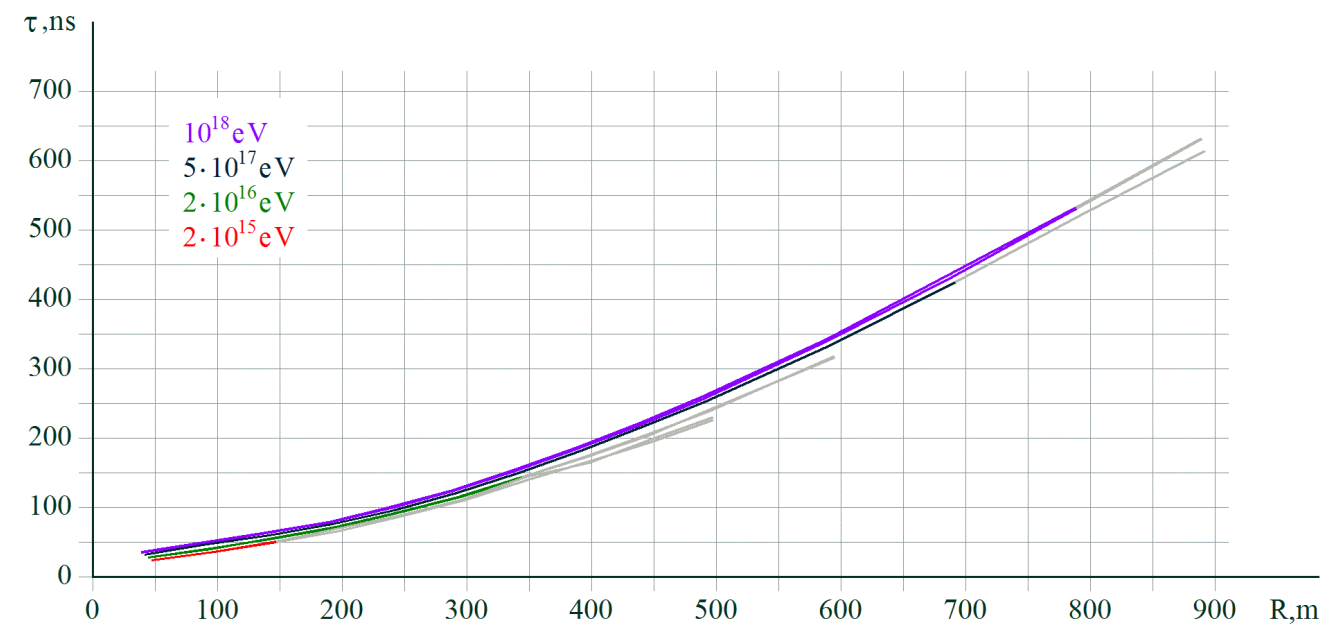

Figure 5: Simulated EAS disk width vs. distance from axis for different $E_{0}$.

Figure 4 shows the dependence of simulated EAS disk width $\left(\tau_{0.8}\right)$ on the distance from the EAS axis for the same energies as Figure 4. The width increases with the distance and it has a weak dependence on $\mathrm{E}_{0}$.

Therefore, from simulation using EHS model, EAS should present itself to the observer as a single disk with statistical variations within it. Disk thickness increases from axis outwards while particle density in the disk decreases from the axis outwards. Large detectable variations should be extremely rare. All these features are found in the majority of events recorded by H10T detector system. 


\section{EAS with Delayed Particles are Multimodal Events}

By the definition of the EAS with delayed particles, the prompt group of particles passing the detectors is followed by the delayed group after some time. The spatial and temporal structure of charged particles in EAS has been studied in thousands of detected events by H10T with nslevel resolution.

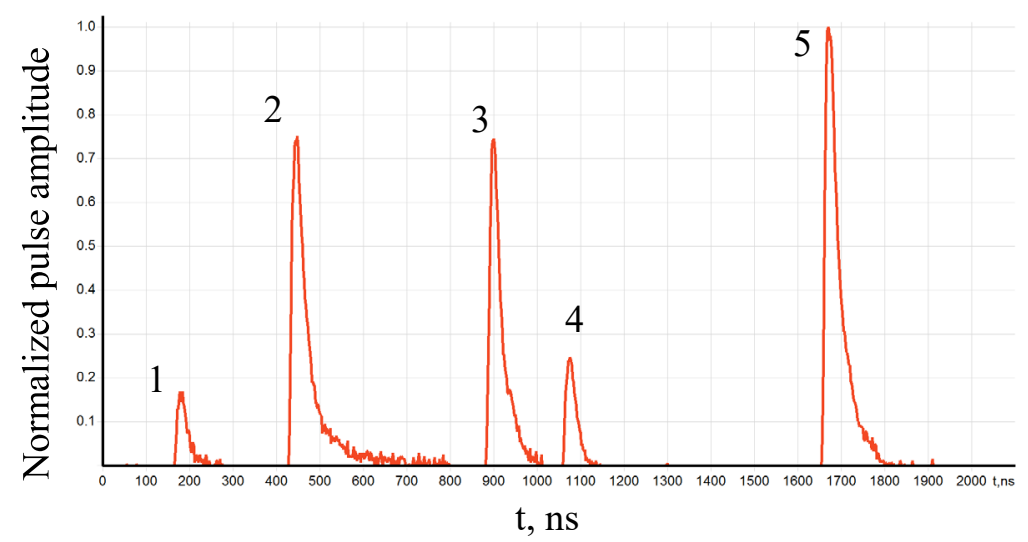

Figure 6: Event from April 6, 2018, as seen by H10T detection point 9. Labels are peak numbers.

For illustration, Figure 6 shows the EAS event registered on April 6, 2019. The EAS axis arrived from Southern direction with the zenith angle of $52^{\circ}$. The figure shows only pulses from detection point 9 that was at the distance $\mathrm{R} \sim 898 \mathrm{~m}$ from EAS axis. Five separate pulses can be clearly distinguished.

Table 3: Detector system acceptance and event rate.

\begin{tabular}{|c|c|c|c|c|c|}
\hline Peak \# & 1 & 2 & 3 & 4 & 5 \\
\hline Delay, ns & 0 & 265 & 720 & 894 & 1493 \\
\hline$\tau_{\mathbf{0 . 8}}, \mathbf{n s}$ & 28 & 34 & 31 & 31 & 35 \\
\hline $\boldsymbol{\rho}_{\mathbf{0 . 8}}, \mathbf{M}^{-\mathbf{2}}$ & 32 & 256 & 187 & 52 & 339 \\
\hline
\end{tabular}

The characteristics of all pulses from Figure 6 are listed in Table 3: the delay of each peak from the first one, the width of each peak $\tau_{0.8}$ and the $\rho_{0.8}$, particle density within the $\tau_{0.8}$.

From the Figure 3 and Table 3 data we can see how EAS from April 6, 2019, is different from the EHS model.

i. There are 5 pulses registered at a detection point. From Figure 2, EHS disk has a uniform structure without breaks and should produce a single pulse.

ii. At $\mathrm{R} \sim 898 \mathrm{~m}$ from axis, the average particle density is $\sim 173$ particles $/ \mathrm{m}^{2}$. The sum is $\sim 566$ particles $/ \mathrm{m}^{2}$. From simulation in Figure 4, at this distance for the EHS with $\mathrm{E}_{0}=10^{18} \mathrm{eV}$ the particle density is below one particle $/ \mathrm{m}^{2}$.

iii. From simulation in Figure 5, the width of the pulse at $\mathrm{R} \sim 898 \mathrm{~m}$ should be approaching $600 \mathrm{~ns}$. The measured widths are between $28 \mathrm{~ns}$ and $35 \mathrm{~ns}$.

Ergo, we can conclude that a registered event with such characteristics can no be described by EHS model. 
The 'delayed particle' notion calls for the existence of two unequal groups of particles prompt and delayed. In EHS, the primary particle gives birth to a single disk of particles. The existence of the 'delayed particle' assumed that unknown physical process separates a delayed group of particles from the main one so that is detected later in time.

Five pulses in Figure 6 indicate the passage of five particle groups though the detector, among which the main group cannot be assigned. Since there are multiple pulses with each pulse having a maximum (or a mode), we shall call such signal a multimodal pulse. An event in which the multimodal pulses have been detected will be called multimodal as well.

\subsection{Spatial and Temporal Characteristics of Bimodal Events}

In the data collected by H10T between Feb $15^{\text {th }}$ to May $12^{\text {th }}$ of 2018 there are 217 events that feature two pulses registered at detection point 9. A signal with two pulses is called bimodal. The location of the axis and the distance $\mathrm{R}$ from it for detection point 9 are determined for each of these 217 events. From each bimodal pulse, the following values were extracted:

- Delay time of the second pulse to the first one

- Particle density corresponding to first and second pulses

- Width of the first and second pulses.

Figure 7 shows the points $(\rho, R)$ that illustrate the distribution of cumulative particle density (e.g. sum for both pulses) for each bimodal pulse vs. the distance to axis R superimposed onto Figure 4 for comparison.

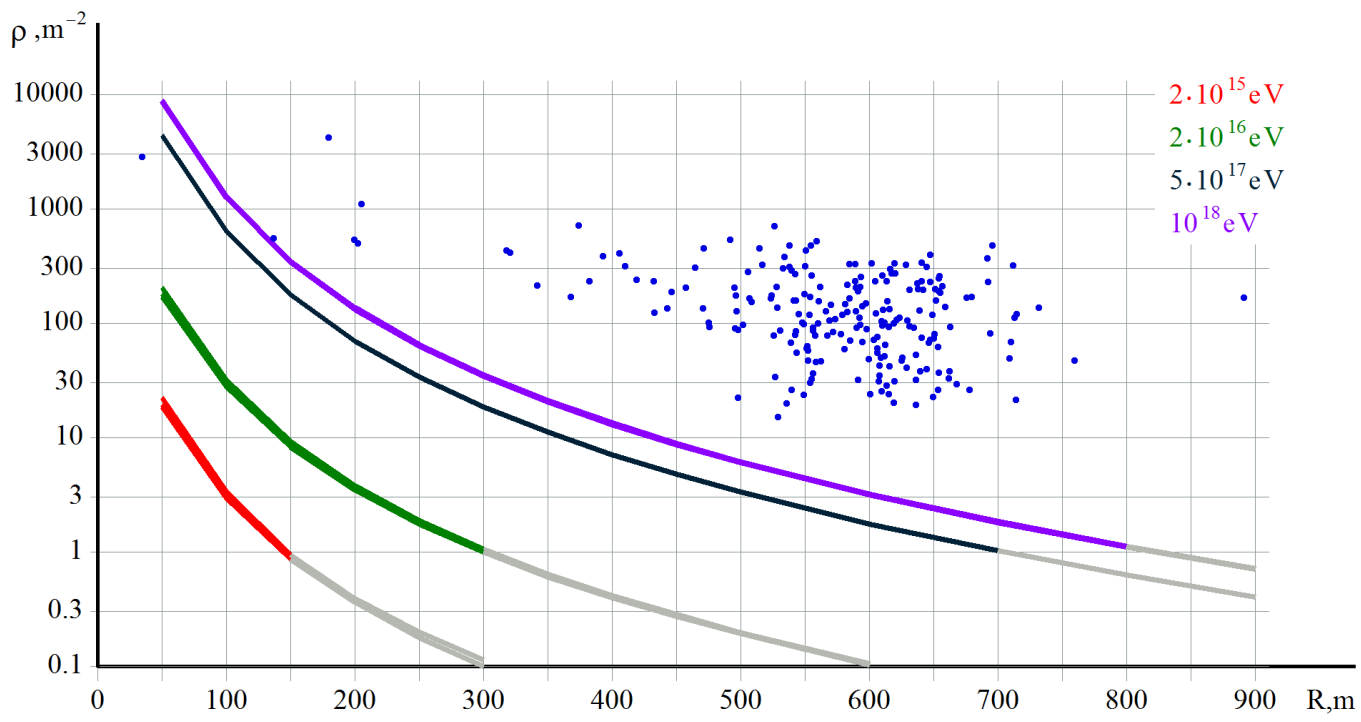

Figure 7: Cumulative particle density for each bimodal pulse vs. distance to EAS axis.

From Figure 7, the detected particle density is much higher than suggested by simulation. To achieve such densities, the energy of the primary causing these events should be drastically higher, causing also much lower detection rate from the H10T acceptance.

Figure 8 shows the $\tau_{0.8}$ for the first pulse (in blue) and second pulse (in red) for the 217 events with bimodal pulses vs. the distance to the EAS axis superimposed onto Figure 5 for comparison. We see that the width of both pulses is much lower from suggested by the simulation. Additionally, there is no pattern in widths of the first and second pulses vs. their order. 


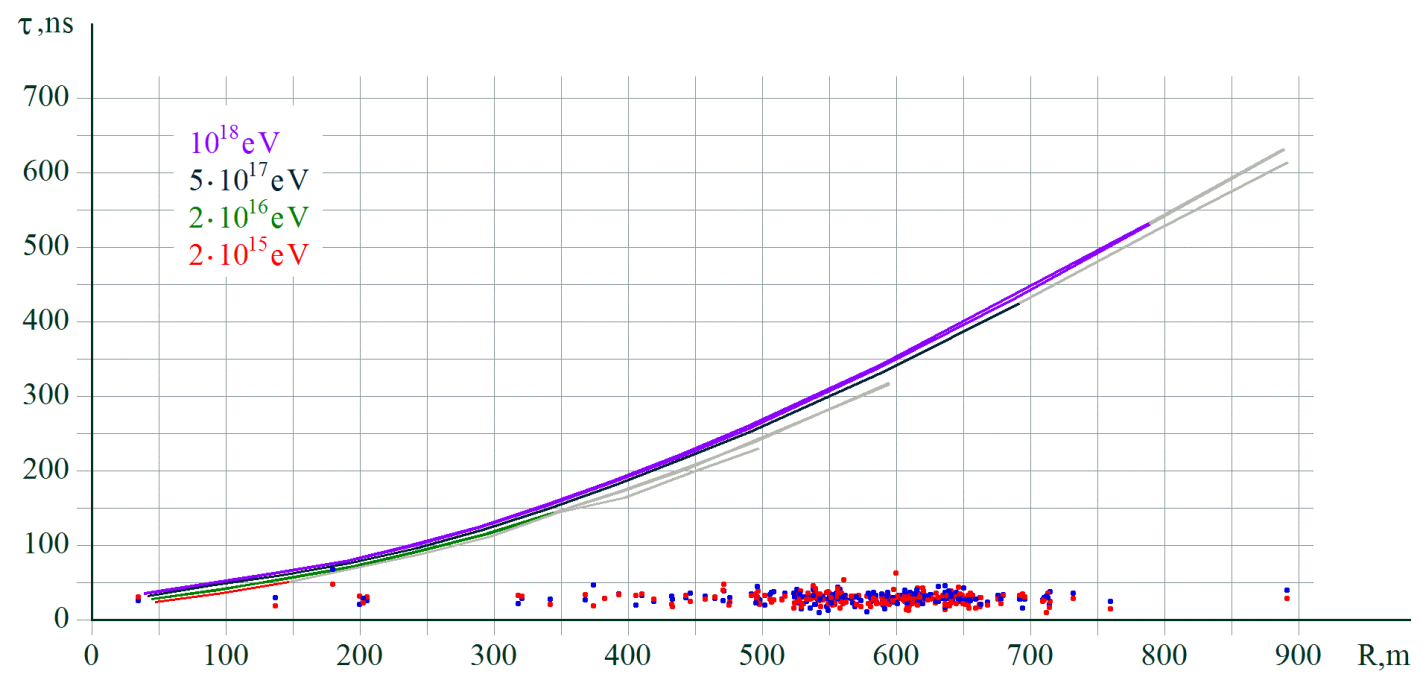

Figure 8: Pulse width $\tau_{0.8}$ for the first pulse (in blue) and second pulse (in red) for the bimodal pulses vs. distance to the EAS axis.

\section{Conclusion}

A total of 217 bimodal events were found in the experimental data collected by H10T experiment from Feb $15^{\text {th }}$ to May $12^{\text {th }}$ of 2018. Each of these events has a bimodal pulse registered at the detection point 9. The first and second pulses in bimodal events are similar to each other. The simulations using CORSIKA package showed that EHS cannot form bimodal pulses.

In those bimodal events, the charged particle density stays steady on the level of 30 to 300 particles $/ \mathrm{m}^{2}$ at the distances of $500-700 \mathrm{~m}$ from the shower axis. The EHS with energy of the primary $10^{18} \mathrm{eV}$, at the distance of $600 \mathrm{~m}$ from the shower axis the average charged particle density is $\sim 3$ particles $/ \mathrm{m}^{2}$. The experimentally observed density will be present in EHS with energy of the primary particle above $10^{19} \mathrm{eV}$, however, EAS with such energy should be detected by H10T once every few years.

In the 217 recorded bimodal events, the width of the pulses at the distances from the shower axis of $400-700 \mathrm{~m}$ is about $20-35 \mathrm{~ns}$. In the simulated EHS at the distance of $600 \mathrm{~m}$ the pulse width is $\sim 350 \mathrm{~ns}$.

These results indicate that the bimodal EAS are not pure electron-hadron showers. Therefore, current analysis methods of EHS, such as using a function of spatial distribution of particles, is only partially applicable to multimodal EAS (also known as EAS with delayed particles). 


\section{References}

[1] J V Jelley and W J Whitehouse. 1953 Proc. Phys. Soc. A 66 (454), 1953

[2] J. Linsley and L. Scarsi. Phys. Rev. 128 (2384), 1962

[3] Baxter A.J., Watson A.A., Wilson J.G. Proc. 9 ICRC. 2 (724), 1965

[4] H. Sakuyama, N. Suzuki, and K. Watanabe. Nuovo Cim. A 78 (147), 1983

[5] Fomin Yu.A., Garipov G.K. et al., Proc. 28 ICRC. 1 (973), 2003

[6] Rashid Beisembaev et al., EPJ Web of Conferences 208 (08008), 2019

[7] G. T. Zatsepin, Proceedings of the USSR Academy of Sciences 67 (993), 1949

[8] D. Heck, J. Knapp, J.N. Capdevielle, G. Schatz, T. Thouw. CORSIKA: A Monte Carlo Code to Simulate Extensive Air Showers, Forschungszentrum Karlsruhe Report FZKA (6019) 